\title{
Multi-View Sketch-based FreeForm Modeling
}

\author{
Florian Levet, Xavier Granier, and Christophe Schlick
}

IPARLA project (INRIA futurs - LaBRI), UMR 5800, Université Bordeaux 1; 351, cours de la Libération 33405 Talence, France

$\{$ levet,granier, schlick\}@labri.fr

\begin{abstract}
For the generation of freeform 3D models, one of the most intuitive solution is to use sketch-based modeling environments. Unfortunately, since the user interface relies upon the analyse of sketches in order to determine which action is requested by the user, the possible amount of different operations can be limited. In this paper, we present a $3 \mathrm{D}$ sketching system based on multiple views. Each view is specialized on a component of the modeling process (like the skeleton, the profile, etc.), and is based on specific sketching interactions. With this approach, an user could improve its understanding of the modeling process and perform a larger range of modeling operations.
\end{abstract}

Key words: Sketch-based 3D Modeling

\section{Introduction}

In order to create simpler interfaces, new approaches for 3D modeling have been developed, based on the human ability to quickly draw a global overview of an object. These approaches are commonly referred as $3 D$ Sketching. Their principle is to infer the shape of a 3D model and add details thanks to different editing operations (e.g., cutting, extrusion), all based on sketched 2D curves.

One of the limitations of these systems is due to their exclusive use of sketches. Since they are only strokes drawn by the user, their shape and the operations that they perform are obviously limited. While classic 3D modelers have dozens of possible operations made available by cascading menus and buttons, each 3D sketching system can only perform few operations based on its analysis of the strokes.

In this paper, we present a new $3 \mathrm{D}$ sketching interface based on multiple views for the creation 3D freeform models. Each view provides is a specialized into a component of the complete modeling process. Since each view has its dedicated sketching interactions, our system allows a larger variety of sketchbased modeling tools compared to existing systems. Our system is based on the following goals: (1) he has to preserve and further develop the sketching interaction of the editing operations for each view, (2) he has to present all the characteristics of the models thanks to the distinct views and, finally, (3) he has to provide an easy switch between the different views.

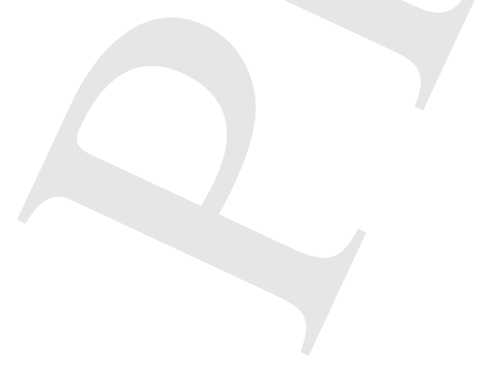




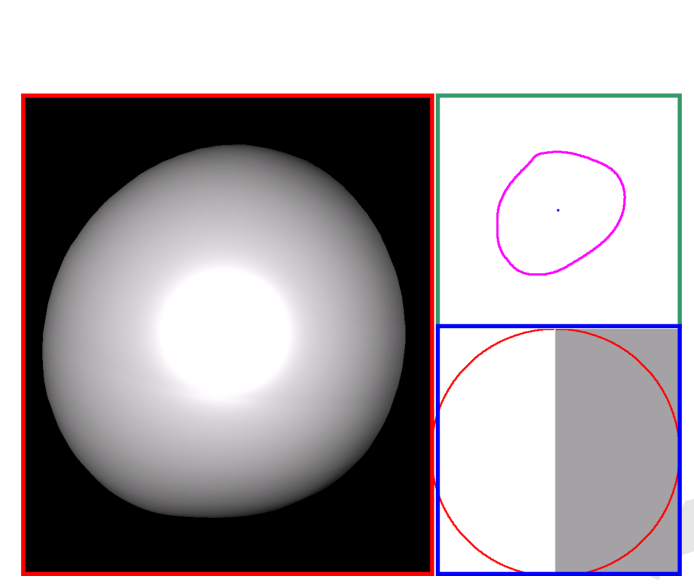

(a)

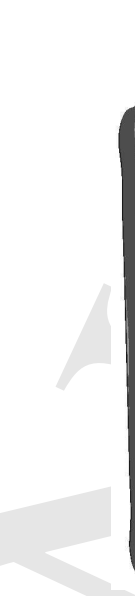

(b)

Fig. 1. (a) Three different views are used in our system: the 3D view (left frame) that shows the 3D object under construction, the skeleton view (upper-right frame) which shows the outline of the object and its skeleton and, the profile view (bottom-right frame) that shows the profile currently used to create the object. (b) A door created with two objects. Their positionning was done in the $3 \mathrm{D}$ view.

\section{Previous Work}

Teddy [3] is the precursor of 3D freeform modeling tools based on gestures and curves. From a sketched 2D silhouette, the system extracts a skeleton and infers the 3D mesh of the object based on a circular profile. Moreover, a gesture grammar converts drawn curves into corresponding modeling operations: extrusion, deformation, cutting, etc. Since the system is totally based on sketches, it is easy to create interesting objects even for non-expert users of 3D modeling softwares. But, since some editing operations are difficult on meshes, many following works are based on alternative geometric representations of their objects. For instance, Owada et. [7] used voxels in order to create internal structures. Many approaches (e.g., $[4,9,8]$ ) use implicit surfaces because of their nice mathematical properties: these systems define the 3D object as a set of implicit primitives stored in a tree structure and with an easy fusion between different primitives.

More recently, some systems $[9,2,6]$ have introduced profile editing for sketchbased modeling. Their goal is to remove the usual blobby aspect of $3 \mathrm{D}$ models created with sketch-based modeling tools. Nevertheless, one main limitation of all these systems is that they only present one single view (or a pop-up window which is closed after the editing operation [6]) during the modeling task. Thus, only a part of the object information is displayed at a given time.

In traditional 3D modeling systems (e.g., 3DS Max, Blender), possible geometry misunderstandings can be solved by using multiple $2 \mathrm{D}$ views of the same scene. Moreover, some editing tasks can be performed more efficiently and accurately on $2 \mathrm{D}$ views. In this paper, we propose to extend this idea to sketching systems. 


\section{3D Sketching with multiple views}

Figure 1 shows a snapshot of our prototype system. Currently, it is based on three different views: the 3D view (see the left frame), the skeleton view (see the upper-right frame) and the profile view (see the bottom-right frame). As said in Section 1, each views is specialized into a component of the modeling process and defines its own sketching interactions. Moreover, their aims are different:

- The 3D view: this is the common view used in standard one-view 3D sketching systems. All the sketching operations related to the 3D mesh of the object (cut, extrusion, etc...) are implemented in this views.

- The skeleton view: this view is related to the skeleton of the object. In this view, users can either select an area on the object by selecting the corresponding region on the skeleton, or modify the extracted skeleton.

- The profile view: in this view, users can change the profile of either the complete object or the currently selected region.

\subsection{The 3D view}

The $3 \mathrm{D}$ view is very similar to the standard one-view of other $3 \mathrm{D}$ sketching systems and, is mainly used to perform three tasks: (i) the visualization of the $3 \mathrm{D}$ objects, (ii) the positionning of these $3 \mathrm{D}$ objects (either by moving them see Figure 1(b) - or by defining a new 2D plane for the silhouette curve) and, (iii) 3D editing of the objects with all the Teddy-like operations such as cutting, extrusion or fusion.

\subsection{The skeleton view}

When the user has finished to sketch the 2D silhouette of an object, this curve is first sampled and, then, the silhouette points are transferred to the skeleton view. Starting from them, a two-step process is used to compute the skeleton. First, the pointset is approximated with a 2D variational implicit surface [10]. The gradient of this implicit surface is then used to determine the vanishing points (points where the gradient drops to zero) that are the skeleton seeds. Since we know the neighboring relations between them, we can easily construct axial edges between neighboring seeds as well as radial edges that link the axial edges to the silhouette curve (see [5] for more details about this technique).

Although this process can be totally automatic, we prefer to let the user influence the creation of the resulting skeleton. Therefore, users are allowed to either move, discard or create skeleton points..

\subsection{The profile view}

All the operations related to the profile curve are defined on the profile view which is the last view provided by our system. More precisely, users have just to draw a $2 \mathrm{D}$ sketch to define a new profile. In our current implementation, only half 


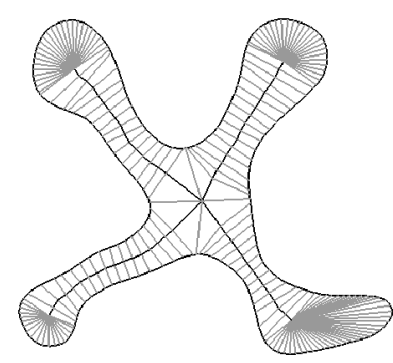

(a)

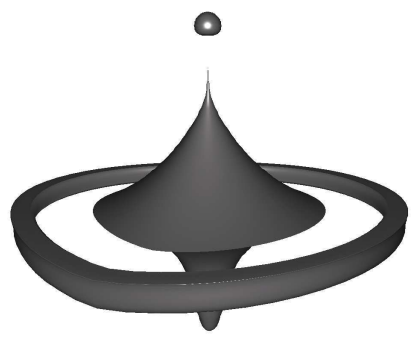

(b)

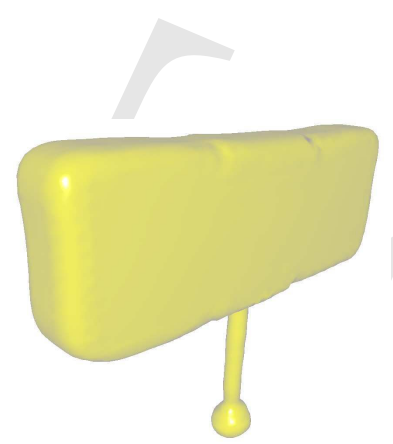

(c)

Fig. 2. (a) Final skeleton with axial edges (in black) and radial edges (in grey). (b) A genus 2 object obtained with only one silhouette and one profile curve. (c) A hammer created with our system.

of the curve has to be drawn and the other half is reconstructed by symmetry [6]. Besides, since users may want to create a 3D blobby object by only sketching a silhouette curve, we have designed a default circular profile curve which is loaded at the beginning of our system session. Once the profile curve has been generated, it is then sampled and the sample points are transferred to the 3D view to be applied on the current selected area. If no local area of the object has been selected (see Section 4), the new profile curve is applied on the whole object. Note that our system allows the definition of disconnected components for the profile curve in order to get objects which genus is more than 0 (see Figure 2(b)).

\section{Interactive Session}

The use of three different views, each one with a specific sketching interaction, leads to a better understanding of the characteristics of the 3D model under construction. Indeed, having all these informations (silhouette curve, profile curve, axial and radial edges) displayed at the same time in the same view would be disturbing for the user. Moreover, some occlusions can occur between these different elements. By having three views, we separate the information and we can display all the elements at the same time.

Note that it is possible to change the position of the three views, as the 3D view is not necessary the more important view for a specific operation. In some case, the user may want to have the skeleton or the profile view as the principal view. So, we offer a straightforward sketch-based interface to swap the position of two different views: this is done by starting a stroke in one view, and finishing it in the other one (see Figure 3).

A usual session of our system starts with the interactive sketching of the silhouette curve. Then, the system determines the seed points of the skeleton, as well as their initial relationship. The user may want to influence the creation of this skeleton, as explained above, or just let the system perform it automatically. 


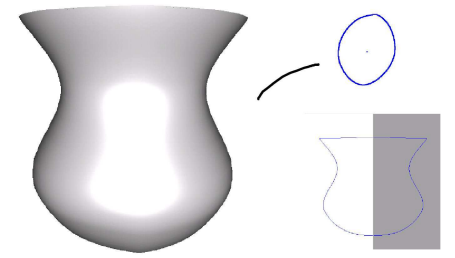

(a)

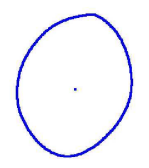

(b)

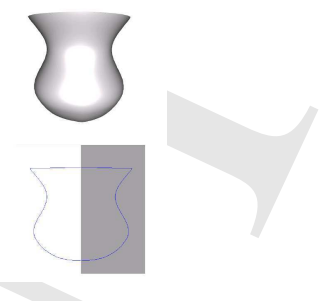

(

Fig. 3. (a) The default setting of the system with the 3D view as the principal view. (b) The user has swapped the skeleton view and the $3 \mathrm{D}$ view by sketching a stroke.

Afterwards, the axial edges and the radial ones are computed from the skeleton and, finally, the system infers the volume of the object by using the profile curve designed in the profile view.

After having defined a first version of the 3D object, the user can still modify its global shape by editing either the silhouette curve, the seed points or the profile curve. This basically redefines the mesh of the 3D object from scratch. For more local editing, we offer another possibility that works by selecting a subset of radial edges and applying a new profile curve on them, which only involves displacement of mesh vertices.

Figure 4 presents an example of such local editing. The selection of a subset of radial edges has been easily performed in the skeleton view since it's a 2D view (see Figure 4(a)). This task would have been painful to realize in the 3D view. Then, a new profile curve is sketched in the profile view, leading to the modification of the corresponding selected points of the mesh (see Figure 4(b)). Since, the three views are displayed, the user can easily understand the effects of this editing operation.

Finally, users can obtain models which have a genus greater than 0 (see Figure 2(b)) by drawing a profile curve with disconnected components. More complex models are easily obtained by combining different primitives as can be seen with the door of Figure 1(b) and the hammer of Figure 2(c).

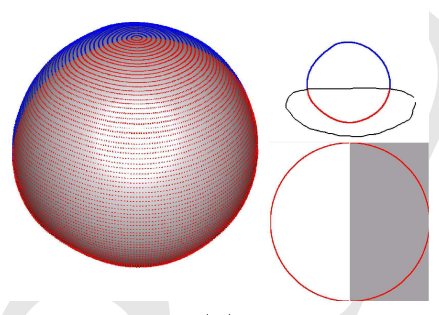

(a)

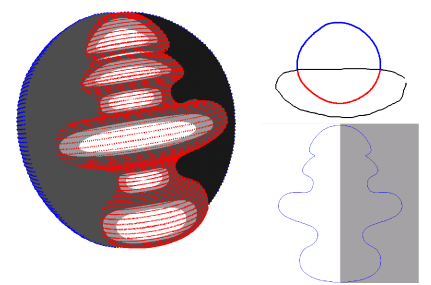

(b)

Fig. 4. (a) A radial edge selection has been done on the skeleton view (the bounding stroke). (b) Selected points have been moved to match the new sketched profile curve. 


\section{Conclusion and future work}

In this paper, we have presented a new $3 \mathrm{D}$ sketching system that uses multiple views, where each one provides some kind of specialization of the complete modeling process. Displaying all the degrees of freedom of a 3D model in a single view can be disturbing and hardly understandable. This is the reason for which we split the display and only present relevant information to each component. However, because all the views are displayed at the same time, our system provides efficient visual clues to the user to understand how the creation of the 3D model is performed. Moreover, each view defines a specific sketching interaction, enabling a much larger variety of editing operations.

Two directions of research are focusing our attention right now. First, we plan to further develop the sketching interface of the already defined views. For instance, we want to offer more interaction in the skeleton view to give a better control over the skeleton computation. Finally, we think that we can use even more views with our system. For instance, we plan to design a specialized view which define a suggestive interface [1]. The aim of this view would be to suggest different results (related to the possible operations) when a user draw a stroke.

\section{References}

1. B. De Araujo and J. Jorge. Blobmaker: Free-form modelling with variational implicit surfaces. In Proc. of Encontro Português de Computação Grafica” (EPCG), pages 17-26, October 2003.

2. J. J. Cherlin, F. Samavati, M. C. Sousa, and J. A. Jorge. Sketch-based modeling with few strokes. In $S C C G$ '05: Proc of the 21st spring conference on Computer graphics, pages 137-145, 2005.

3. T. Igarashi, S. Matsuoka, and H. Tanaka. Teddy: a sketching interface for 3d freeform design. In Proc. of ACM SIGGRAPH '99, pages 409-416, 1999.

4. O. Karpenko, J. Hughes, and R. Raskar. Free-form Sketching with Variational Implicit Surfaces. Computer Graphics Forum (Proc. of Annual Eurographics Conference 2002), 21(3):585-594, 2002.

5. Florian Levet and Xavier Granier. Improved skeleton extraction and surface generation for sketch-based modeling. In GI (Graphics Interface), 2007.

6. Florian Levet, Xavier Granier, and Christophe Schlick. 3d sketching with profile curves. In International Symposium on Smart Graphics, 2006.

7. S. Owada, F. Nielsen, K. Nakazawa, and T. Igarashi. A Sketching Interface for Modeling the Internal Structures of 3D Shapes. In Proc. of Smart Graphics, pages 49-57, July 2003.

8. R. Schmidt, B. Wyvill, M.C. Sousa, and J.A. Jorge. ShapeShop: Sketch-Based Solid Modeling with BlobTrees. In Eurographics Workshop on Sketch-Based Interfaces and Modeling, pages 53-62, 2005.

9. C.-L. Tai, H. Zhang, and C.-K. Fong. Prototype Modeling from Sketched Silhouettes based on Convolution Surfaces. Computer Graphics Forum, 23(1):71-83, 2004.

10. G. Turk and J. O'Brien. Variational implicit surfaces. Technical Report GITGVU-99-15, Georgia Institute of Technology, 1998.

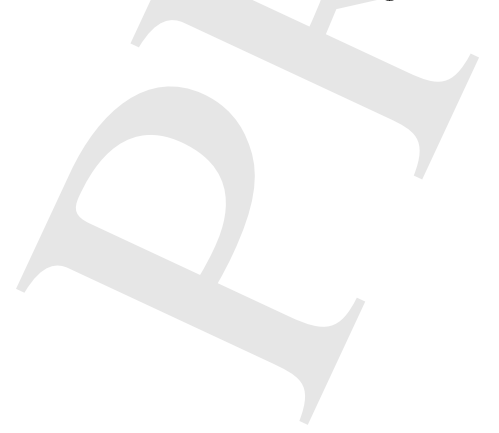

